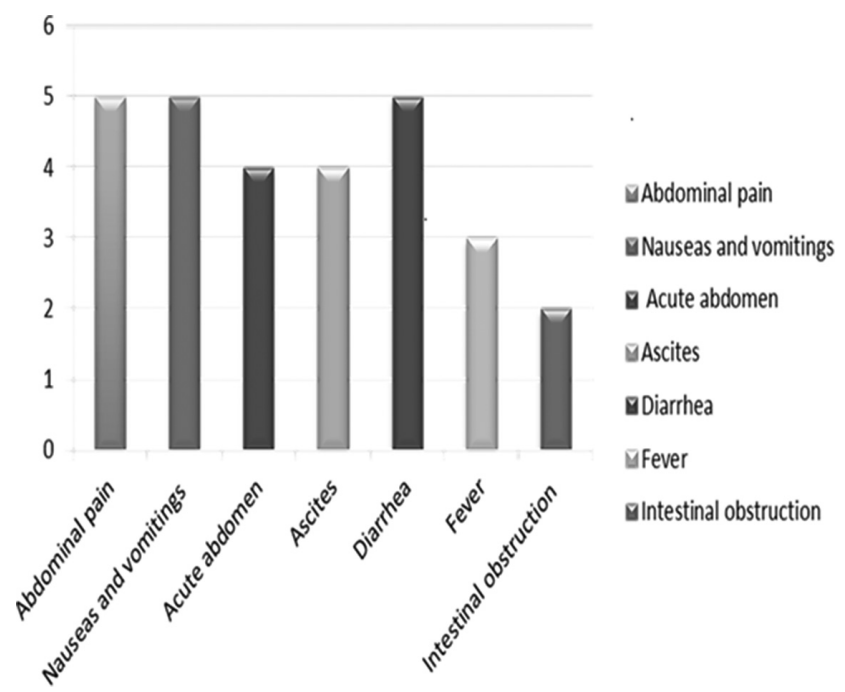

Abstract 229 Figure 1

and bowel dilatation (96\%). All patients received induction with high dose pulse methylprednisone $1000 \mathrm{mg}$ IV for three days followed by prednisone at a dose of $1 \mathrm{mg} / \mathrm{kg} /$ day. Subsequently, Mycophenolate mofetil $1000 \mathrm{mg}$ twice a day was added in the regimen for remission. Only 1 patient developed ileocecal preforation but survived the condition after undergoing laparotomy and ileostomy. Relapses were uncommon ( $25 \%$ ) and managed with optimisation of immunosuppressive regimen.

Results

Conclusions Mycophenolate mofetil (MMF) appears promising as an agent of remission induction and maintenance in patients with lupus enteritis.

\section{META ANALYSIS OF LOW-DOSE ASPIRIN IN REDUCING RISK OF ATHEROSCLEROSIS CARDIOVASCULAR DISEASE IN SYSTEMIC LUPUS ERYTHEMATOSUS PATIENTS}

${ }^{1}$ RA Nugraha* ${ }^{1} \mathrm{M}$ Jonatan, ${ }^{1} \mathrm{FF}$ Alkaff, ${ }^{2} \mathrm{~A}$ Awalia, ${ }^{3} \mathrm{R}$ Yudiwati. ${ }^{1}$ Universitas Airlangga, Medicine, Surabaya, Indonesia; ${ }^{2}$ Internal Medicine - Dr. Soetomo General Hospital, Rheumatology, Surabaya, Indonesia; ${ }^{3}$ Universitas Airlangga, Biomedics and Biomolecular Medicine, Surabaya, Indonesia

\subsection{6/lupus-2017-000215.230}

Background and aims Atherosclerosis Cardiovascular Disease (ASCVD) contributes to higher morbidity and mortality in SLE patients. Aspirin is known to be associated with a decrease in the incidence of thromboembolic events in general population, but its potential benefit in SLE patients has not yet been investigated. Thus, aim of this study is to discover whether aspirin has a significant protective effect on the risk of ASCVD in SLE patients.

Methods Twelve RCT studies identified from the Medline, Embase and Cochrane databases were selected with available individual patient-level data, reporting the use of low-dose aspirin in SLE patients. The primary outcome was the incidence of ASCVD in SLE patients treated with low-dose aspirin compared to those not treated with low-dose aspirin. The secondary outcome was frequency and duration of SLE exacerbation during a mean 7 years follow-up.

Results Pooled effect estimates were obtained using a randomeffects model. Pooled Hazard Ratios (HRs) and 95\% CIs were calculated using Bayesian hierarchical models. We pooled data from 2.135 subjects with 364 ASCVD events during a mean 7 years follow-up. Subgroup analysis showed a protective effect of low-dose aspirin against ASCVD, including CHD, TIA, stroke, and PAD (HR: 0.43 [95\%CI: 0.20-0.93]) but not for SLE exacerbation (HR: 0.49 [95\%CI: 0.22-1.11]).

Conclusions Meta-analysis shows significant decreased of ASCVD events by low dose aspirin among SLE patients. Lowdose Aspirin are considered safe and may be beneficial for thromboprophylaxis. Moreover, bigger studies are needed to provide a better recommendation for clinicians in using lowdose Aspirin in SLE patients.

\section{SYSTEMIC LUPUS ERYTHEMATOSUS PRESENTING AS OCULOMOTOR DISTURBANCE}

${ }^{1}$ E Ogawa*, 'T Hoshiyama, ${ }^{1,2} \mathrm{~J}$ Okada. 'Kitasato University Medical Centre, Department of Rheumatology and infectious diseases, Kitamoto, Japan; ${ }^{2}$ Kitasato Junior College of Health and Hygienic Sciences, Applied Clinical Dietetics, Minami-uonuma, Japan

\subsection{6/lupus-2017-000215.231}

Background and aims Central nervous system (CNS) involvement is one of the serious complication of systemic lupus erythematosus (SLE). We will show a representative case of SLE to suggest that the delay in the initial treatment will bring aftereffects.

Methods We described a 31-year-old Japanese female patient who was diagnosed with SLE at age $16 \mathrm{y} / \mathrm{o}$. SLE has been treated with prednisone for 14 years. The patient with dysarthria, double vision and vertigo visited a nearby hospital. The first brain MRI scan revealed the absence of infarction. She received administration of intravenous immunoglobulin with a primary diagnosis of Fisher syndrome. One day later, she developed facial nerve palsy, truncal ataxia and lowerextremity weakness. The second brain MRI scan revealed the presence of pontine infarction and she was transferred to our hospital. Anti-Sm antibody and anti-ribosomal-P antibody in sera were positive. On the other hand, lupus anticoagulant and B2GPI cardiolipin antibody were negative. Anti-neuronal antibody in CSF was positive. For such occasions, the chief pathogenesis of this case is considered thrombosis of cerebral artery than CNS angiitis. Treatment was started with pulses of methylprednisolone and pulses of cyclophosphamide, followed by oral prednisone.

Results After intensive medical therapy, dysarthria and facial nerve palsy were improved. However, double vision and truncal ataxia has remained.

Conclusions If effective treatment has not been performed early like this case, it is likely to leave physical impairment. Although oculomotor disturbance is rare complication of SLE, SLE patients must be examined and treated considering that this complication will develop as a part of neuropsychiatricSLE. 